\title{
ON THE GIBBS' PHENOMENON IN A CERTAIN EIGENFUNCTION SERIES
}

LUNA I. MISHOE

I. Introduction. In a paper by B. Friedman and Luna Mishoe [1] it is shown that the series,

$$
\sum_{-\infty}^{\infty} a_{n} u_{n}(x)
$$

where

$$
a_{n}=\left(\int_{0}^{1} u_{n}(\xi) B^{*} v_{n}(\xi) d \xi\right)^{-1} \int_{0}^{1} f(\xi) B^{*} v_{n}(\xi) d \xi ; u_{n}(x)
$$

being eigenfunctions or nonzero solutions of the system

$$
u^{\prime \prime}+q(x) u+\lambda\left[p(x) u-u^{\prime}\right]=0, \quad u(0)=u(1)=0
$$

and $v_{n}(x)$ being eigenfunctions of the system adjoint to (2), where $B^{*}=d / d x+p(x) ;$ converges to;

$$
1 / 2[f(x+)+f(x-)]+c \exp \int_{0}^{x} p d t
$$

provided $f(x)$ is of bounded variation, and $p(x)$ possesses a continuous second derivative, and $q(x)$ is continuous on $(0,1)$. In the expression (3) above

$$
c=-1 / 2\left[f(0+)+f(1-) \exp -\int_{0}^{1} p d t\right] .
$$

In this paper the following theorem is proved:

Theorem. The partial sum $\sigma_{n}(x) \equiv \sum_{k=-n}^{n} a_{k} u_{k}(x)$ exhibits Gibbs' phenomenon for the function $g(x) \equiv f(x)+c \exp \int_{0}^{x} p d t$ whenever the Fourier partial sum $S_{n}(x) \equiv \sum_{k=-n}^{n} \exp (2 k \pi i x) \int_{0}^{1} f(\xi) \exp (-2 k \pi i \xi) d \xi$ exhibits Gibbs' phenomenon for $f(x)$.

II. A relationship between $\sigma_{n}(x)$ and $S_{n}(x)$. In [1] it is shown that:

$$
\begin{aligned}
u_{n}(x) & =\lambda_{n}^{-1}\left[\exp \left(\lambda_{n} x-\int_{0}^{x} p d t\right)-\exp \left(\int_{0}^{x} p d t\right)\right]+O\left(\frac{\left(1_{1}\right.}{\lambda_{n}^{2}}\right), \\
B^{*} v_{n}(x) & =\exp \left(-\lambda_{n} x+\int_{0}^{x} p d t\right)+O \frac{\left(e^{-\lambda n x}\right)}{\lambda_{n}}
\end{aligned}
$$

Received by the editors April 29, 1957. 
where $O(1)$ means a bounded function as $|\lambda| \rightarrow \infty$.

In [2] it is shown that:

$$
\lambda_{n}=2 n \pi i+\int_{0}^{1} p d t+O\left(\frac{1}{n}\right) .
$$

From (5) and (6) it follows that

$$
\begin{aligned}
\int_{0}^{1} u_{n}(\xi) B^{*} v_{n}(\xi) d \xi= & \int_{0}^{1} \frac{1}{\lambda} d \xi+\int_{0}^{1} O\left(\frac{1}{\lambda}\right) d \xi \\
& -\int_{0}^{1} \exp \left(-\lambda_{n} \xi+\int_{0}^{\xi} p d t\right) d \xi=\frac{1+O\left(1 / \lambda_{n}\right)}{\lambda_{n}}
\end{aligned}
$$

and consequently

$$
\left[\int_{0}^{1} u_{n}(\xi) B^{*} v_{n}(\xi) d \xi\right]^{-1}=\frac{\lambda_{n}}{1+O(1 / n)}=\lambda_{n}\left[1+O\left(\frac{1}{n}\right)\right]
$$

for large $n$.

Now in (5) if we use the fact that $\exp \left(-\int_{0}^{x} p d t\right)=1+O(1)$ we have

$$
u_{n}(x)=\lambda_{n}^{-1}\left[e^{\lambda_{n} x}+O(1)+O\left(\frac{1}{n}\right)\right]
$$

and

$$
\int_{0}^{1} f(\xi) B^{*} v_{n}(\xi) d \xi=\int_{0}^{1} f(\xi) e^{-\lambda_{n} \xi} d \xi+O(1) .
$$

From (8), (9), and (10) we obtain

$$
\begin{aligned}
a_{n} u_{n}(x) & \equiv u_{n}(x)\left[\int_{0}^{1} u_{n}(\xi) B^{*} v_{n}(\xi) d \xi\right]^{-1} \int_{0}^{1} f(\xi) B^{*} v_{n}(\xi) d \xi \\
& =e^{\lambda n x} \int_{0}^{1} f(\xi) e^{-\lambda n \xi} d \xi+O(1) .
\end{aligned}
$$

Using the value of $\lambda_{n}$ as given in (6) we have

$$
e^{\lambda n x}=e^{2 n \pi i x} \cdot \exp \left(2 x \int_{0}^{1} p d t+O\left(\frac{x}{n}\right)\right)=e^{2 n \pi i x}+O(1)
$$

and consequently

$$
a_{n} u_{n}(x)=e^{2 n \pi i x} \int_{0}^{1} f(\xi) e^{-2 n \pi i \xi} d \xi+E_{n}(x),
$$

where 


$$
E_{n}(x)=O(1)+O(1 / n)
$$

And we have,

$$
\sigma_{n}(x) \equiv \sum_{k=-n}^{n} a_{k} u_{k}(x)=\sum_{k=-n}^{n} e^{2 k \pi i x} \int_{0}^{1} f(\xi) e^{-2 k \pi i \xi} d \xi+\sum_{k=-n}^{n} E_{k}(x)
$$

or $\sigma_{n}(x)=S_{n}(x)+T_{n}(x)$ where $S_{n}(x)$ is the Fourier partial sum of $f(x)$ and $T_{n}(x) \equiv \sum_{k=-n}^{n} E_{k}(x)$. This is our desired relationship.

III. Uniform convergence of the series $\sum_{-\infty}^{\infty} E_{n}(x)$ in $(0,1)$. Since [1] $\sum_{-\infty}^{\infty} a_{n} u_{n}(x)=1[f(x+)+f(x-)] / 2+c \exp \int_{0}^{x} p d t$; it follows that

$$
\begin{aligned}
\sum_{-\infty}^{\infty}\left[e^{2 n \pi i x} \int_{0}^{1} f(\xi) e^{-2 n \pi i \xi} d \xi+E_{n}(x)\right] \\
=1 / 2[f(x+)+f(x-)]+c \exp \left(\int_{0}^{x} p d t\right)
\end{aligned}
$$

and since

$$
\sum e^{2 n \pi i x} \int_{0}^{1} f(\xi) e^{-2 n \pi i \xi} d \xi=1 / 2[f(x+)+f(x-)] \quad \text { for } 0<x<1
$$

we have

$$
\begin{aligned}
\sum_{-\infty}^{\infty}\left[e^{2 n \pi i x} \int_{0}^{1} f(\xi) e^{-2 n \pi i \xi} d \xi+E_{n}(x)\right] \\
=c \exp \left(\int_{0}^{x} p d t\right)+\sum_{-\infty}^{\infty} e^{2 n \pi i x} \int_{0}^{1} f(\xi) e^{-2 n \pi i \xi} d \xi
\end{aligned}
$$

and it follows that

$$
\sum_{-\infty}^{\infty} E_{n}(x)=c \exp \left(\int_{0}^{x} p d t\right)
$$

Now since $p(x)$ possess a continuous second derivative on $(0,1)$ it follows that $c \exp \left(\int_{0}^{x} p d t\right)$ is both continuous and of bounded variation on $(0,1)$ and therefore may be expanded into a Fourier series which converges uniformly to $c \exp \left(\int_{0}^{x} p d t\right)$ for $0<x<1$. From this we conclude that

$$
\sum_{-\infty}^{\infty} E_{n}(x)=c \exp \left(\int_{0}^{x} p d t\right) \quad \text { uniformly for } 0<x<1 .
$$


IV. Gibbs' Phenomenon. Let $f(x)$ possess a finite discontinuity at $x=a_{1}$ and let $\alpha_{n}$ and $\beta_{n}$ be the abscissa and ordinate respectively of the first maxima (or minima) of $y=S_{n}(x)$ to the left or right of $x=a_{1}$, then as

$$
n \rightarrow \infty, \quad \alpha_{n} \rightarrow a_{1}
$$

But on the left of $a_{1}$

$$
\beta_{n} \rightarrow f\left(a_{1}-0\right)+\left[\frac{f\left(a_{1}+0\right)-f\left(a_{1}-0\right)}{\pi} \int_{\pi}^{\infty} \frac{\sin x}{x} d x\right]
$$

and on the right of $x=a_{1}$

$$
\beta_{n} \rightarrow f\left(a_{1}+0\right)-\left[\frac{f\left(a_{1}+0\right)-f\left(a_{1}-0\right)}{\pi} \int_{\pi}^{\infty} \frac{\sin x}{x} d x\right] .
$$

This is Gibbs' Phenomenon for the partial sum $S_{n}(x)$ of the Fourier Series of $f(x)$.

Since $T_{n}(x)=c \exp \left(\int_{0}^{x} p d t\right)$ uniformly in $(0,1)$ as $n \rightarrow \infty$, this partial sum does not exhibit Gibbs' Phenomenon.

Note that corresponding to the maxima (or minima) $\left(\alpha_{n}, \beta_{n}\right)$ on the curve, $y=S_{n}(x)$ there is a point $\left(\alpha_{n}, \beta_{n}^{1}\right)$ on the curve $y^{1}=\sigma_{n}(x)$ such that

$$
\beta_{n}^{1}=\beta_{n}+c \exp \left(\int_{0}^{\alpha_{n}} p d t\right)
$$

Now as $n \rightarrow \infty, \alpha_{n} \rightarrow a_{1}$

$$
\begin{aligned}
\beta_{n}^{1} \rightarrow f\left(a_{1}-0\right)+ & {\left[\frac{f\left(a_{1}+0\right)-f\left(a_{1}-0\right)}{\pi} \int_{\pi}^{\infty} \frac{\sin x}{x} d x\right] } \\
& +c \exp \left(\int_{0}^{a_{1}} p d t\right)
\end{aligned}
$$

on the left of $x=a_{1}$ and

$$
\begin{aligned}
\beta_{n}^{1} \rightarrow f\left(a_{1}+0\right)- & {\left[\frac{f\left(a_{1}+0\right)-f\left(a_{1}-0\right)}{\pi} \int_{\pi}^{\infty} \frac{\sin x}{x} d x\right] } \\
& +c \exp \left(\int_{0}^{a_{1}} p d t\right)
\end{aligned}
$$

on the right of $x=a_{1}$. Or as $n \rightarrow \infty$

$$
\alpha_{n} \rightarrow a_{1}
$$


and since $f\left(a_{1}+0\right)-f\left(a_{1}-0\right)=g\left(a_{1}+0\right)-g\left(a_{1}-0\right)$ where

$$
g(x)=f(x)+c \exp \left(\int_{0}^{x} p d t\right)
$$

we have

$$
\beta_{n}^{1} \rightarrow g\left(a_{1}-0\right)+\left[\frac{g\left(a_{1}+0\right)-g\left(a_{1}-0\right)}{\pi} \int_{\pi}^{\infty} \frac{\sin x}{x} d x\right]
$$

on the left of $x=a_{1}$ and

$$
\beta_{n}^{1} \rightarrow g(a+0)-\left[\frac{g\left(a_{1}+0\right)-g\left(a_{1}-0\right)}{\pi} \int_{\pi}^{\infty} \frac{\sin x}{x} d x\right]
$$

on the right of $x=a_{1}$, where $g(x)=f(x)+c \exp \left(\int_{0}^{x} p d t\right)$. The results in (20), (21), and (22) shows clearly that $y^{1}=\sigma_{n}(x)$ exhibits Gibbs' Phenomenon for

$$
g(x)=f(x) c \exp \left(\int_{0}^{x} p d t\right)
$$

wherever $y=S_{n}(x)$ exhibits this Phenomenon for $f(x)$.

\section{REFERENCES}

1. Bernard Friedman and L. I. Mishoe, Eigenfunction expansions associated with a non-self-adjoint differential equation, Pacific J. Math. vol. 6, no. 2 (1956).

2. L. I. Mishoe and G. C. Ford, On the uniform convergence of a certain eigenunction series, Pacific J. Math. vol. 6, no. 2 (1956).

Morgan State College 